\title{
Complex modulus of round window membrane over auditory frequencies in normal and otitis media chinchilla ears
}

\section{Xuelin Wang, Don Nakmali and Rong Z. Gan*}

School of Aerospace and Mechanical Engineering and

Bioengineering Center,

University of Oklahoma,

Norman, OK 73019, USA

Fax: (405)325-1088

Email: xlwang@ou.edu

Email: donnakmali@ou.edu

Email: rgan@ou.edu

*Corresponding author

\begin{abstract}
To better reveal the mechanical properties of round window membrane (RWM) in normal and pathological ears, the complex modulus of chinchilla RWM was determined by measuring its dynamic behaviour together with the finite element simulation. The acute otitis media (AOM) was created by transbullar injection of Haemophilus influenzae in chinchillas and RWM specimens in AOM ears were prepared four days post inoculation. Vibration of the RWM induced by coil-magnet force stimulation was measured by laser Doppler vibrometry over frequencies of $0.2-8 \mathrm{kHz}$. A finite element model-based inverse-problem solving method was used to determine the complex modulus of each RWM specimen in the frequency domain. Experimental results of the AOM ears indicated that the resonance frequency decreased by $13.94 \%$ compared with the normal ears and the mean storage modulus and loss modulus were decreased by $65 \%$ and $32 \%$, respectively.
\end{abstract}

Keywords: round window membrane; RWM; complex modulus; viscoelasticity; otitis media; finite element model.

Reference to this paper should be made as follows: Wang, X., Nakmali, D. and Gan, R.Z. (2015) 'Complex modulus of round window membrane over auditory frequencies in normal and otitis media chinchilla ears', Int. J. Experimental and Computational Biomechanics, Vol. 3, No. 1, pp.27-44.

\footnotetext{
Biographical notes: Xuelin Wang received his $\mathrm{PhD}$ in Mechanical Engineering at Huazhong University of Science and Technology, Wuhan, China in 1998. He is currently a Research Scientist in the School of Aerospace and Mechanical Engineering and OU Bioengineering Center, University of Oklahoma, Norman, OK, USA.

Don Nakmali received his MS in Bioengineering at the University of Oklahoma in 2012. He is currently a Biomedical Research Engineer in Biomedical Engineering Lab at OU Bioengineering Center, Norman and Hough Ear Institute, Oklahoma City.
} 
Rong Z. Gan received her $\mathrm{PhD}$ in Biomechanical Engineering at the University of Memphis in 1992. She is the Charles E. Foster Chair and Professor of Biomedical and Mechanical Engineering in the School of Aerospace and Mechanical Engineering and OU Bioengineering Center, University of Oklahoma. She is on the editorial board of International Journal of Experimental and Computational Biomechanics.

\section{Introduction}

Round window membrane (RWM) covers the round window, one of the two openings into cochlea from the middle ear. The role of RWM is to release mechanical energy and/or conduct sound vibration to the scala tympani of the cochlea (Wever and Lawrence, 1948; Goycoolea and Lundman, 1997). The compliance of RWM affects its ability to be displaced by, or to displace, cochlear perilymph.

Acute otitis media (AOM) is the most commonly diagnosed disease in young children, which is usually caused by bacterial or viral invasion. AOM induces morphological changes of the middle ear tissues, including the tympanic membrane (TM), stapedial annular ligament and RWM, which have been observed in patients and experimental animal models (Sahni et al., 1987; Goycoolea, 1995; Schachern et al., 2008; Yoon and Hellstrom, 2002). Recently, an AOM model of guinea pig induced by Streptococcus pneumonia was created and the changes of RWM mechanical properties were reported by Gan et al. (2013). However, the biomechanical changes of RWM in other animal models of normal and pathological ears have rarely been reported. In fact, the mechanical properties of soft tissues in the middle ear serve as important measures for transmission of sound into cochlea.

Mechanical properties of human RWM were first reported by Ishii et al. (1995) using a testing apparatus specially designed for small and delicate biological specimens. The mechanical properties of the RWM were obtained under quasi-static loading conditions. Recently, Zhang and Gan (2013) developed a method to quantify the dynamic properties of the human RWM over the auditory frequency range. Vibration of the RWM in response to acoustic pressure stimulation was measured by laser Doppler vibrometry (LDV), and the dynamic properties of the membrane were derived by finite element-based inverse-problem solving method. The mean thickness of human RWM is approximately $70 \mu \mathrm{m}$ (Goycoolea and Lundman, 1997). Another measurement method using the electro-magnetic force stimulation and LDV has been used to characterise the dynamic properties of the guinea pig RWM in normal and AOM ears (Gan et al., 2013). The RWM of guinea pig resembles that of the human, although there is interspecies variation in size and thickness (Saber et al., 2009).

Chinchilla is a popular animal model for auditory research because the range of hearing $(20 \mathrm{~Hz}$ to $30 \mathrm{kHz}$ ) is close to that of humans (Browning and Granich, 1978; Vrettakos et al., 1988). The thickness of chinchilla RWM is about 11-14 $\mu \mathrm{m}$ (Goycoolea et al., 1988). However, due to the different ultrastructure of the RWM among animals, no prior studies have documented if such difference affects dynamic properties of the RWM in AOM ears. In this study, the electro-magnetic force stimulation associated with the inverse-problem solving technique was used to quantify the mechanical properties of the RWM over the auditory frequencies in normal and AOM chinchillas. 


\section{Methods}

\subsection{Specimen preparation}

\subsubsection{Creation of AOM model}

Eleven adult chinchillas of mixed gender weighing between 600 and $780 \mathrm{~g}$ were utilised in this study. The study protocol was approved by the Institutional Animal Care and Use Committee of the University of Oklahoma and met the guidelines of the National Institutes of Health. Prior to bacterial challenge, all animals were free of middle ear disease as evaluated by otoscopic examination.

Chinchillas were randomly divided into control and AOM groups. The control group had three animals for six healthy RWM samples, and the AOM group included four animals for eight AOM samples. Additionally, specimens from two animals of four ears of each group were used for histology studies. The AOM model was produced by transbullar injection of Haemophilus influenzae 86-028NP suspension into both ears following the procedure reported by Guan et al. (2014). Briefly, under general anaesthesia [ketamine $(10 \mathrm{mg} / \mathrm{kg})$ and xylazine $(2 \mathrm{mg} / \mathrm{kg})], 0.3 \mathrm{ml}$ bacterial suspension containing 3,000 CFU was injected into the superior bulla bilaterally using a $1 \mathrm{cc}$ syringe with a 26 gauge needle. After the challenge dose was administrated, otoscopic examination was performed daily.

Four days post inoculation, the animals were anesthetised with ketamine $(40 \mathrm{mg} / \mathrm{kg})$ and xylazine $(10 \mathrm{mg} / \mathrm{kg})$. The TM was examined under otoscopy to determine the colour of the TM as well as the presence of the effusion in the middle ear. In control ears, the TM was visualised microscopically to confirm normal colour without an effusion in the middle ear cavity. Then, the animals were euthanised by an overdose of ketamine $(100 \mathrm{mg} / \mathrm{kg})$ and xylazine $(20 \mathrm{mg} / \mathrm{kg})$ and the bullae were harvested to prepare the RWM samples for mechanical tests and histology studies.

\subsubsection{RWM specimen preparation for dynamic testing}

Six specimens from the control group and seven specimens from the AOM group were used for vibration test. One AOM sample was ruptured in the preparation. The bulla was trimmed by removing the bony wall and cut into a block containing the malleus-incus complex, stapes, cochlea and promontory. Subsequently, a cut at the inferior edge of the footplate created a flat surface to open the view of RWM from the scala tympani. The bone near the round window niche was removed to expose the RWM from both sides: the middle ear or lateral side and the cochlear or medial side. Specimens were examined under a microscope to verify that the RWM was not damaged during the preparation. A magnetic microbead shaped like a disc $250 \mu \mathrm{m}$ in diameter and $125 \mu \mathrm{m}$ in thickness with a mass of $0.03 \mathrm{mg}$ (Fair-Rite Products Corp., NY) was placed on the medial side at the centre of the RWM. The bead was held in place by the moistened mucosal tissue on the RWM. Figure 1 shows the photograph of a RWM specimen with the magnet bead attached. The RWM has an elliptical shape with the long axis (a) and short axis (b). Tables 1 and 2 list the dimensions measured from each specimen using the image analysis software (ImageJ) in control and AOM ears, respectively. The average long and short axes for control RWM were 1.43 and $1.0 \mathrm{~mm}$, respectively; the average long and short axes for AOM ears were 1.46 and $1.04 \mathrm{~mm}$, respectively. 
Figure 1 Enlarged RWM specimen image with the magnetic bead placed at the centre of the RWM (see online version for colours)

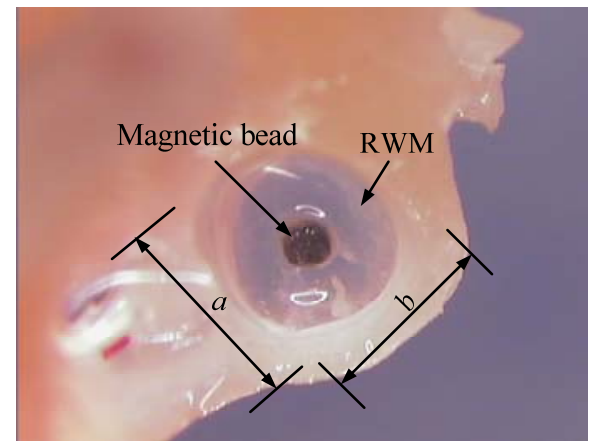

Table 1 The dimensions, resonance frequency $f_{n}$ and vibration amplification ratio $R$ of RWM specimens in control group

\begin{tabular}{lccccccc}
\hline Sample & Con-1 & Con-2 & Con-3 & Con-4 & Con-5 & Con-6 & Mean \pm SD \\
\hline$a(\mathrm{~mm})$ & 1.44 & 1.38 & 1.52 & 1.40 & 1.40 & 1.41 & $1.43 \pm 0.05$ \\
$b(\mathrm{~mm})$ & 1.00 & 0.82 & 1.08 & 1.00 & 1.12 & 1.02 & $1.00 \pm 0.10$ \\
$f_{n}(\mathrm{~Hz})$ & 1,600 & 1,490 & 1,340 & 1,490 & 1,450 & 1,500 & $1,478 \pm 84$ \\
$R$ & 8.10 & 6.00 & 6.40 & 6.30 & 6.70 & 10.30 & $7.30 \pm 1.64$ \\
$E_{0}(\mathrm{MPa})$ & 18.50 & 12.10 & 15.30 & 16.80 & 17.50 & 17.60 & $16.30 \pm 2.32$ \\
$E_{1}(\mathrm{MPa})$ & 8.10 & 7.80 & 10.0 & 10.30 & 10.10 & 7.00 & $8.88 \pm 1.42$ \\
$\tau_{1}(\mu \mathrm{s})$ & 28 & 30 & 29 & 30 & 30 & 27 & $29.0 \pm 1.26$ \\
\hline
\end{tabular}

Note: $E_{0}, E_{1}$ and $\tau_{1}$ are the viscoelastic parameters of RWM specimens derived from FE modelling by the inverse-problem solving method.

Table 2 The dimensions, resonance frequency $f_{n}$ and vibration amplification ratio $R$ of RWM specimens in AOM group

\begin{tabular}{lcccccccc}
\hline Sample & $O M-1$ & $O M-2$ & $O M-3$ & $O M-4$ & $O M-5$ & $O M-6$ & OM-7 & Mean $\pm S D$ \\
\hline$a(\mathrm{~mm})$ & 1.44 & 1.38 & 1.42 & 1.50 & 1.62 & 1.36 & 1.50 & $1.46 \pm 0.09$ \\
$b(\mathrm{~mm})$ & 1.06 & 1.00 & 1.04 & 1.12 & 0.96 & 1.12 & 1.00 & $1.04 \pm 0.08$ \\
$f_{n}(\mathrm{~Hz})$ & 1,340 & 1,340 & 1,510 & 1,130 & 1,060 & 1,190 & 1,340 & $1,273 \pm 154$ \\
$R$ & 16.50 & 11.00 & 9.50 & 6.30 & 8.90 & 10.00 & 5.70 & $9.70 \pm 3.57$ \\
$E_{0}(\mathrm{MPa})$ & 6.50 & 5.85 & 7.55 & 5.30 & 3.65 & 5.50 & 6.05 & $5.77 \pm 1.20$ \\
$E_{1}(\mathrm{MPa})$ & 2.00 & 2.60 & 3.10 & 3.75 & 1.95 & 2.45 & 4.66 & $2.93 \pm 0.99$ \\
$\tau_{1}(\mu \mathrm{s})$ & 25 & 26 & 27 & 29 & 27 & 27 & 30 & $27.28 \pm 1.70$ \\
\hline
\end{tabular}

Note: $E_{0}, E_{1}$ and $\tau_{1}$ are the viscoelastic parameters of RWM specimens derived from FE modelling by the inverse-problem solving method.

\subsection{Experimental setup}

The experimental setup was similar to that used for guinea pig studies by Gan et al. (2013). Figure 2 shows a schematic diagram of the experimental setup and indicates that the vibration of the magnet at the centre of RWM was measured by LDV along the 
vertical direction. Briefly, the electromagnetic coil made from the wire (AWG 30) of 500 turns and the high $\mu$ steel core (Carpenter Technologies Inc., IN) had the inductance of $2.41 \mathrm{mH}$ and resistance of $3.9 \mathrm{Ohm}$. The coil was placed directly under the RWM to vibrate the magnetic bead attached to the membrane. The distance between the coil and bead was maintained at $1 \mathrm{~mm}$. This distance was confirmed under a microscope to ensure that no movement of the bead occurred secondary to the static attraction force between the coil and the magnet bead. The coil was driven by the dynamic signal generator/analyser (DSA, HP35670A, Agilent Tech., CO) and the power amplifier (B\&K 2718, Denmark). An AC current of $16.7 \mathrm{~mA}$ was delivered to the coil sweeping from 0.2 to $8.0 \mathrm{kHz}$. The vibration of the RWM induced by electromagnetic force stimulation was measured using the LDV (HLV-1000, Polytec, Inc., Irvine, CA). The vibration amplitude of the RWM was directly calculated from the voltage output of the LDV velocity decoder.

Figure 2 The schematic of experiment setup for dynamic test on the RWM specimen (see online version for colours)

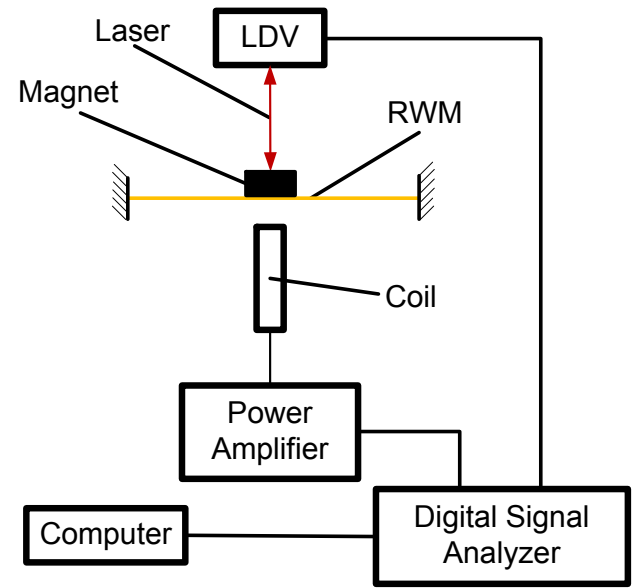

The specimens were preconditioned before tests following the procedure described by Gan et al. (2013). Because of the extremely small size and fixed bony boundary, the RWM specimen was difficult to conduct standard preconditioning in a material testing system. The cyclic hydraulic pressure was applied onto the specimen surface by using saline solution in a $1 \mathrm{cc}$ syringe. The peak pressure was estimated around $10 \mathrm{~Pa}$. Movement of the RWM was observed under a surgical microscope. This process was repeated for five cycles to reach the stabilised state for specimen. Note that the RWM specimen was maintained under moist conditions by spraying saline solution onto the middle ear side surface. 


\subsection{Inverse-problem solving method}

\subsubsection{Finite element model of dynamic test}

The finite element (FE) model-based inverse-problem solving method was used to derive the complex modulus for each RWM sample from the displacement-frequency response curves.

Each RWM specimen was modelled as an elliptic membrane of variable thickness with clamped boundary. The long axis a and short axis b values listed in Tables 1 and 2 were used for building the geometry of each model. The thickness $h$ of the RWM in the FE model was based on measurements of histological section images. Figure 3 shows the typical histological sections of RWM obtained from a control ear and an AOM ear. The thickness of RWM varies from the centre to the edge. We selected the centre and two other locations along axis $\mathrm{a}$ and $\mathrm{b}$ to measure the thickness on histological sections. The measurements showed that the RWM of the AOM ear was thicker than that of control ear at all the locations. For control ears the average thickness of the RWM varied from $10 \mu \mathrm{m}$ at the centre to $11.25 \mu \mathrm{m}$ at the edge; for AOM ears the average thickness of the RWM changed from $15.25 \mu \mathrm{m}$ at the centre to $17 \mu \mathrm{m}$ at the edge. The magnetic microbead attached to the RWM was modelled as an elastic disc with diameter of $250 \mu \mathrm{m}$ and thickness of $125 \mu \mathrm{m}$. The contact between the bead and RWM was modelled without slip.

Figure 3 The histological section images of the RWM, (A) a histological section image in control ear, (B) a histological section image of the RWM in AOM ear (see online version for colours)

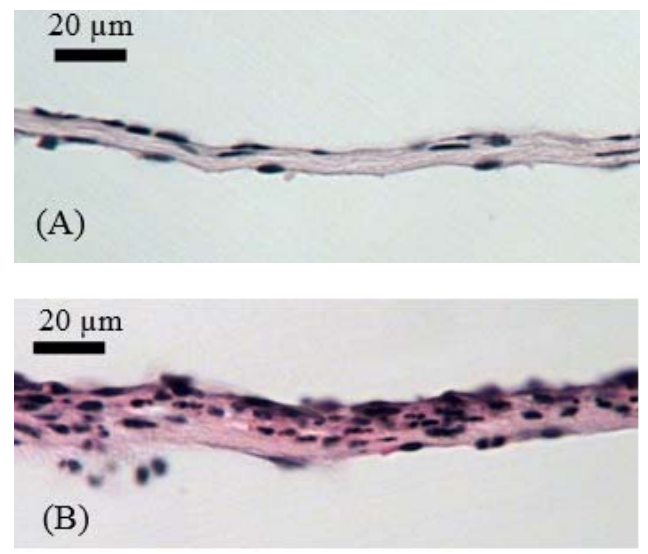

Six FE models of normal RWM and seven models of AOM specimens were generated. Figure 4 shows the FE model of a RWM sample for dynamic simulation with the magnetic bead attached. The RWM was meshed by 8,400 hexahedral elements (type Solid 185 in ANSYS) and a convergence analysis was performed, which showed that the element number was adequate to reach accurate results. The density of the RWM was assumed as $\rho=1,200 \mathrm{~kg} / \mathrm{m}^{3}$, and passion's ratio was $v=0.3$. The microbead was meshed by 6,000 hexahedral elements (type Solid 45 ). The density and elastic modulus of the bead was set as $5,000 \mathrm{~kg} / \mathrm{m}^{3}$ and $210 \mathrm{GPa}$, respectively (Gan et al., 2013). 
Figure 4 The FE models of dynamic experiments on RWM specimens with the bead attached at the centre (see online version for colours)

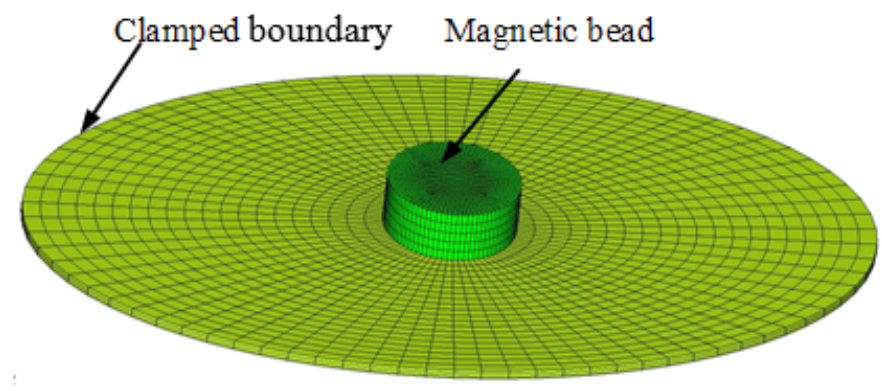

The driving force of $0.03 \mu \mathrm{N}$ applied onto the magnet surface was calculated through the electromagnetic analysis in ANSYS (Ethiraj, 2003) and was validated on aluminium foil test (Gan et al., 2013). The model-derived displacement-frequency curve was compared with the experimental curve for each specimen. The material parameters were then iteratively altered until a good match with the experimental displacement-frequency response was obtained. All RWM specimens were considered to be homogeneous isotropic materials, and the material properties are described in the next section.

\subsubsection{Material constitutive law for RWM specimen}

Because of the composition and structure, biological soft tissues behave mechanically as a viscoelastic solid. The dynamic behaviour of soft tissues in the middle ear is usually time or frequency dependent. The generalised Maxwell model (Machiraju et al., 2006) was used to describe the viscoelastic behaviour of RWM. The relaxation modulus of RWM is expressed as

$$
E(t)=E_{0}+\sum_{i=1}^{n} E_{i} \exp \left(-\frac{t}{\tau_{i}}\right)
$$

where $E_{i}$ is the relaxed elastic modulus of the $i^{\text {th }}$ spring, $\tau_{i}$ is the relaxation time of the $i^{\text {th }}$ dashpot. For harmonic analysis of viscoelastic solid, the applied stress and the resulting strain are not in phase and $E(t)$ can be converted into complex modulus $E^{*}$ in the frequency domain as:

$$
E^{*}(f)=E^{\prime}(f)+i E^{\prime \prime}(f)
$$

where $E^{\prime}(f)$ is the storage modulus, $E^{\prime \prime}(f)$ is the loss modulus, $f$ is the frequency. For estimation of the viscoelastic constants of the RWM, we used $n=1$ in equation (1) in this study, and $E^{\prime}(f)$ and $E^{\prime \prime}(f)$ can be expressed as:

$$
\begin{aligned}
& E^{\prime}(f)=E_{0}+E_{1} \tau_{1}^{2}(2 \pi f)^{2} /\left(1+\tau_{1}^{2}(2 \pi f)^{2}\right) \\
& E^{\prime \prime}(f)=2 \pi E_{1} \tau_{1} f /\left(1+\tau_{1}^{2}(2 \pi f)^{2}\right) \\
& \eta(f)=\tan \delta=E^{\prime \prime}(f) / E^{\prime}(f)
\end{aligned}
$$


where $\delta$ is the phase angle of the complex modulus, $\eta(f)$ is the loss factor.

\section{Results}

\subsection{Thickness change of RWM in AOM ear}

Thickness of the RWM is a key parameter to characterise its mechanical properties. We examined the histological section images of the control and AOM ears, and Figure 3 displays the typical histologic images of RWMs. Note that only partial section images were shown in Figures 3(A) and 3(B).

The thickness was measured in four specimens for each group (control and AOM). A significant difference was observed in the AOM RWM compared with the normal RWM. The average thickness of RWM in normal ears was about $11.0 \mu \mathrm{m}$, and that of AOM ears was $16.7 \mu \mathrm{m}$. The thickness of RWM in AOM ears was increased by $51 \%$ compared with the normal. The thickened RWM as shown in Figure 3(B) was mainly caused by infiltration of the inflammatory cells. The middle fibrous layer of the RWM was also hypertrophic in AOM ears.

\subsection{Experimental measurement data and modelling results}

Figure 5 shows the displacement amplitude - frequency curves measured from six control RWM specimens [Figure 5(a)] and seven AOM specimens [Figure 5(b)]. In control group, each specimen had a prominent displacement peak at a resonance frequency between 1,340 and $1,600 \mathrm{~Hz}$. The resonance frequency $f_{n}$ and displacement amplification ratio $R$, which is defined as the ratio of maximum displacement at $f_{n}$ to the static displacement, are listed in Table 1 for each RWM specimen. In Figure 5(a), the mean resonance frequency was $1,478 \pm 84 \mathrm{~Hz}$. The ratio $R$ ranged between 6.3 and 10.3 with a mean value of 7.30. In AOM group, the resonance frequency ranged from 1,060 to $1,510 \mathrm{~Hz}$. Table 2 lists the resonance frequency and the amplification ratio $R$ of each RWM specimen. The mean resonance frequency was $1,272 \pm 154 \mathrm{~Hz}$ for AOM samples, which was lower than the resonance frequency measured from control samples. The displacement amplification ratio $R$ was ranged between 5.7 and 16.5 with a mean value of 9.7 for AOM samples.

FE models were created to simulate the dynamic experiments of the RWM specimens in control and AOM groups. As an example, Figure 6 shows the results from one specimen in each group [control in Figure 6(a); AOM in Figure 6(b)]. As shown in this figure, the displacement amplitude-frequency curves derived from the FE models (dashed lines) and measured from the experiments (solid lines) match well in the resonance frequency and peak amplitude. Specimen Con-6 had experimental resonance frequency at $1,500 \mathrm{~Hz}$. The resonance frequency of the corresponding FE model was also at 1,500 $\mathrm{Hz}$. Similarly, specimen OM-6 had experimental resonance frequency at $1,190 \mathrm{~Hz}$, while the corresponding model resulted in a same resonance frequency as the experimental result. 
Figure 5 The displacement-frequency curves measured from the experiments over the frequency range of $0.2-8.0 \mathrm{kHz}$, (a) six control RWM samples, (b) seven AOM samples (see online version for colours)

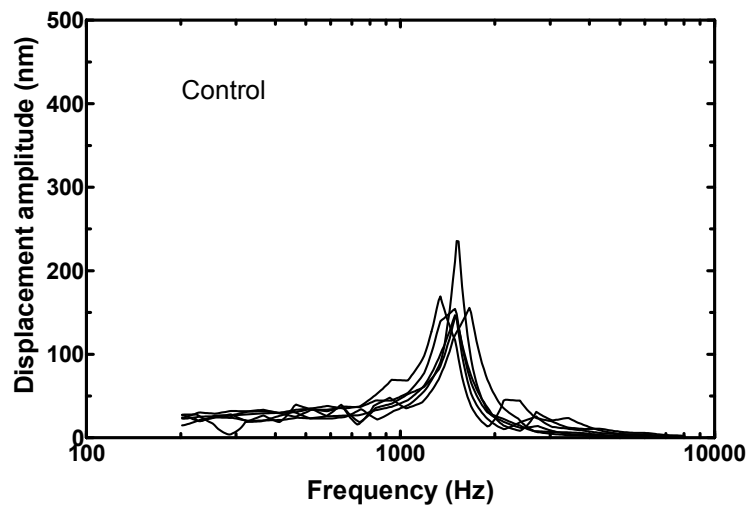

(a)

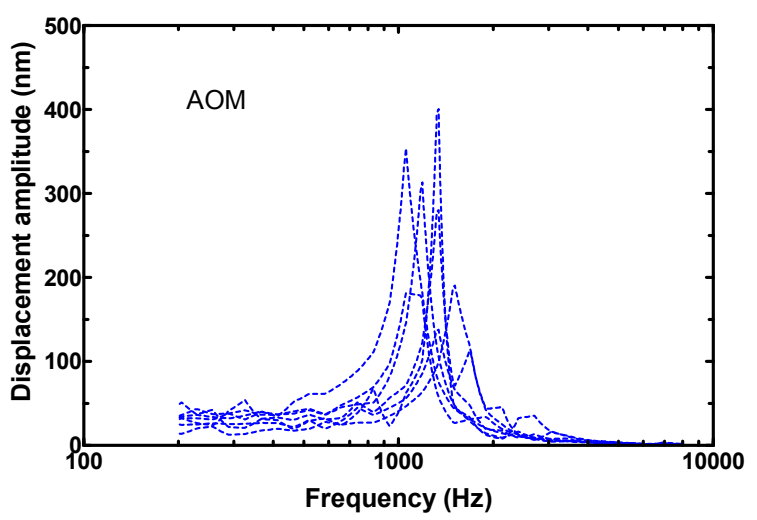

(b)

\subsection{Dynamic properties of RWM in normal and AOM ears}

Three parameters $E_{0}, E_{1}$, and $\tau_{1}$ for each RWM specimen in control and AOM groups were determined through the inverse-problem solving with the FE model, and results are listed in Tables 1 and 2. In control group, the value of $E_{0}$ ranged from 12.10 to $18.50 \mathrm{MPa}$, while $E_{1}$ ranged from 7.00 to $10.30 \mathrm{MPa}$. In the AOM group, the value of $E_{0}$ ranged from 3.65 to $7.55 \mathrm{MPa}$, while $E_{1}$ ranged from 1.95 to $4.66 \mathrm{MPa}$. There were some differences between the specimens for each parameter, which may be caused by individual variations between RWM specimens (physiological conditions, geometric dimensions). 
Figure 6 The FE modelling results of displacement amplitude (dashed lines) from RWM models in comparison with the corresponding experimental curves (solid lines), (a) normal specimen Con-6, (b) AOM specimen OM-6 (see online version for colours)

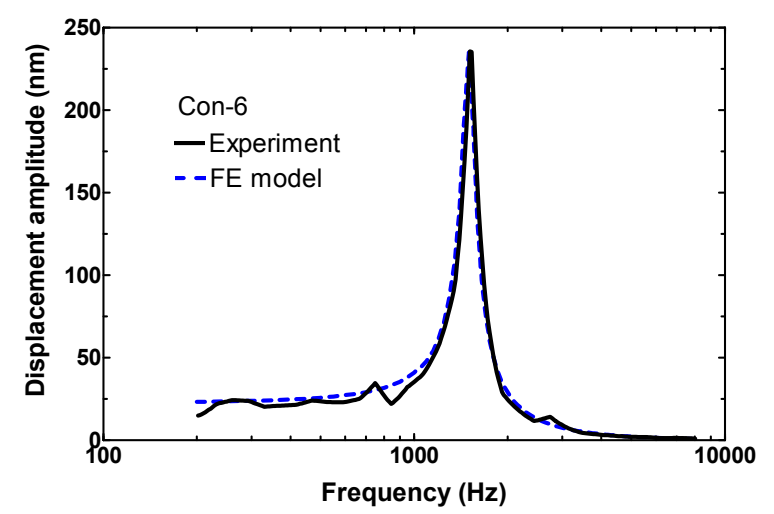

(a)

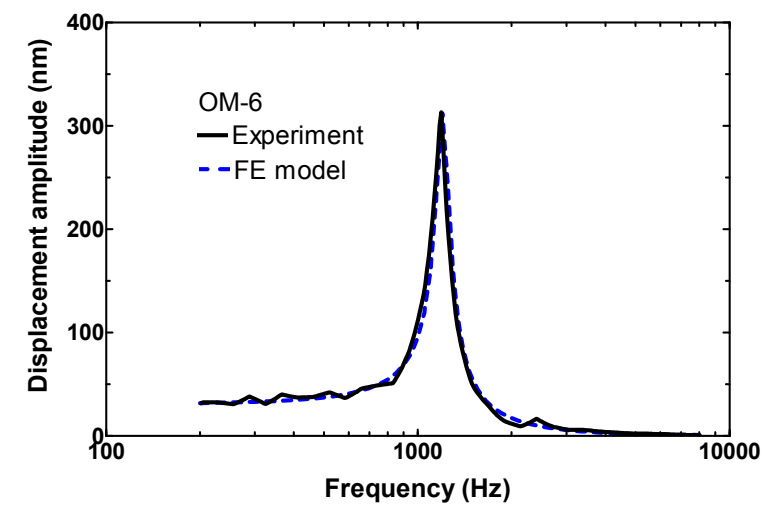

(b)

Based on parameters $E_{0}, E_{1}$ and $\tau_{1}$ obtained from each RWM specimen, the storage modulus $E^{\prime}$ and loss modulus $E^{\prime \prime}$ of each specimen were calculated. Figure 7 shows $E^{\prime}$ and $E^{\prime \prime}$ curves obtained from all control and AOM specimens across the frequency range of 0.2 to $8 \mathrm{kHz}$. Figure 7(a) shows that the storage modulus increased with frequency for all control specimens. The largest storage modulus values were $18.51 \mathrm{MPa}$ at $0.2 \mathrm{kHz}$ (Con-1), and 24.52 MPa at $8 \mathrm{kHz}$ (Con-5). As shown in Figure 7(b), the loss modulus increased with the increase of frequency and reached its maximum value around 5.0-6.0 kHz. The largest loss modulus was $5.15 \mathrm{MPa}$ at $5.5 \mathrm{kHz}$ (Con-5). Variation in both storage and loss modulus for RWM in AOM ears shared similar patterns with that in control ears as shown in Figures 7(c) and 7(d). The largest storage modulus values in the $\mathrm{AOM}$ ears were $7.56 \mathrm{MPa}$ at $0.2 \mathrm{kHz}$, and $9.96 \mathrm{MPa}$ at $8 \mathrm{kHz}(\mathrm{OM}-3)$. The largest loss modulus was $0.175 \mathrm{MPa}$ at $0.2 \mathrm{kHz}$ and $2.15 \mathrm{MPa}$ at $8.0 \mathrm{kHz}(\mathrm{OM}-7)$. The smallest loss modulus was $0.063 \mathrm{MPa}$ at $0.2 \mathrm{kHz}(\mathrm{OM}-1)$ and $0.93 \mathrm{MPa}$ at $8.0 \mathrm{kHz}(\mathrm{OM}-5)$. 
Figure 7 The complex modulus ( $E^{\prime}$ and $\left.E^{\prime \prime}\right)$ determined from six RWM specimens in control ears (a and b) and seven RWM specimens in AOM ears (c and d) over the frequency range of $0.2-8 \mathrm{kHz}$, (a) storage modulus $E^{\prime}$ of the normal RWM, (b) loss modulus $E^{\prime \prime}$ of the normal RWM, (c) storage modulus $E^{\prime}$ of the infectious RWM, (d) loss modulus $E^{\prime \prime}$ of the infectious RWM

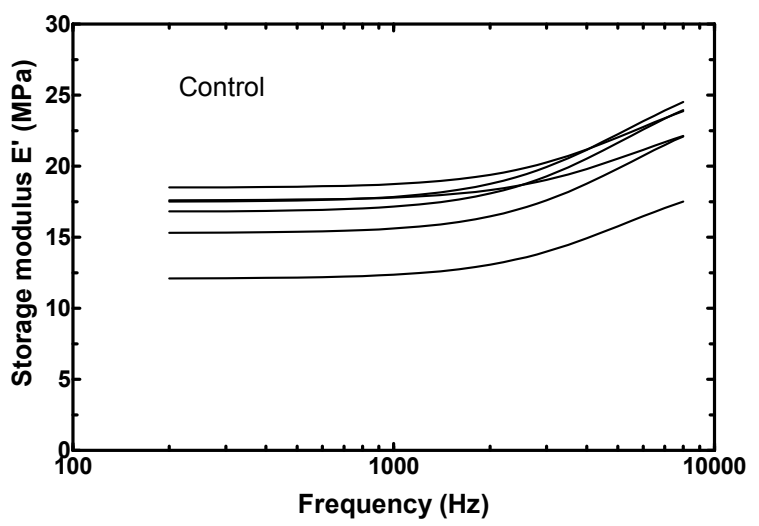

(a)

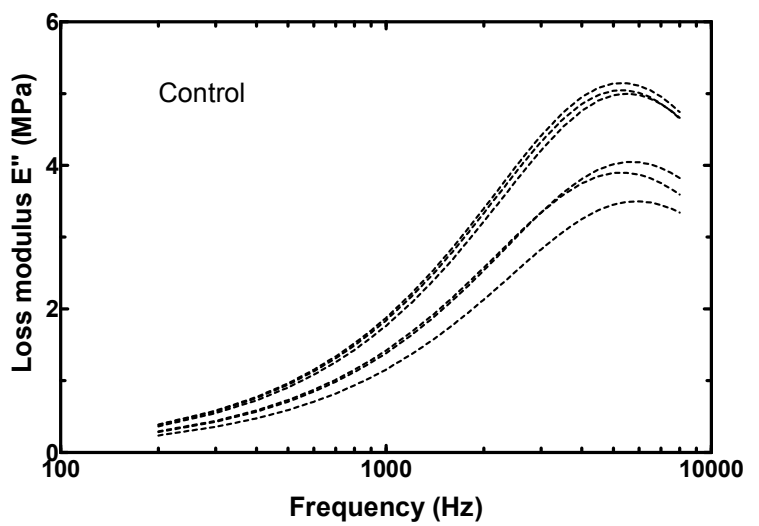

(b)

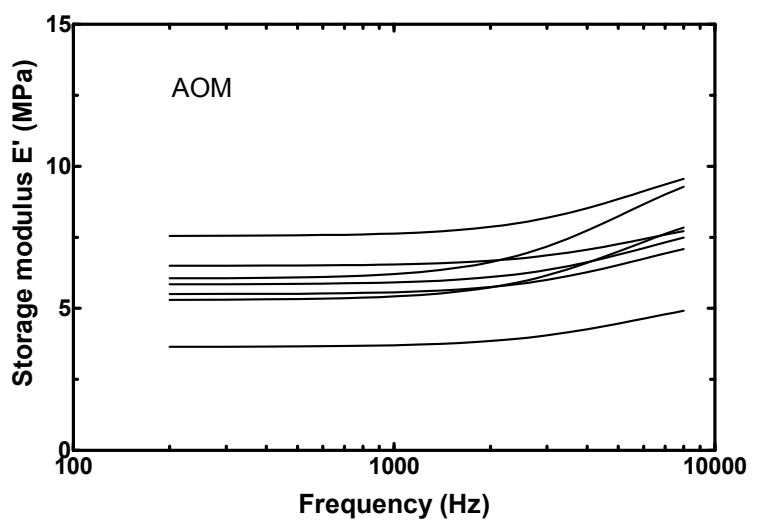

(c) 
Figure 7 The complex modulus ( $E^{\prime}$ and $\left.E^{\prime \prime}\right)$ determined from six RWM specimens in control ears ( $a$ and $b$ ) and seven RWM specimens in AOM ears (c and d) over the frequency range of $0.2-8 \mathrm{kHz}$, (a) storage modulus $E^{\prime}$ of the normal RWM, (b) loss modulus $E^{\prime \prime}$ of the normal RWM, (c) storage modulus $E^{\prime}$ of the infectious RWM, (d) loss modulus $E^{\prime \prime}$ of the infectious RWM (continued)

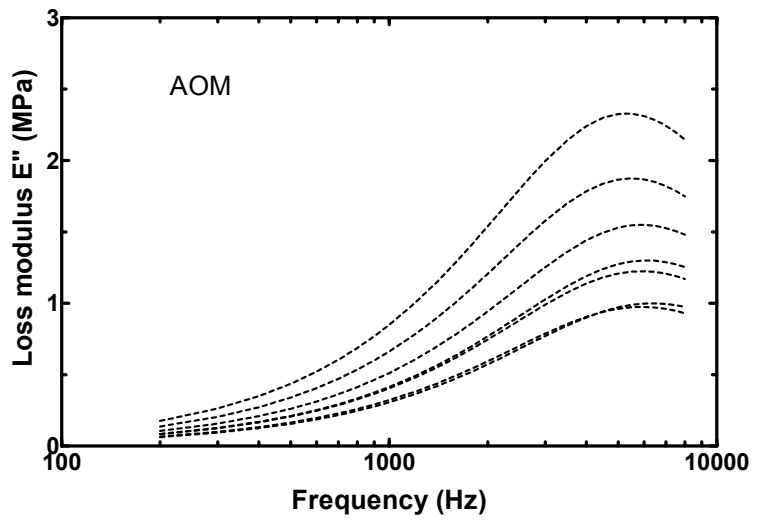

(d)

Mean values of the complex modulus with SD obtained from the six specimens in control group and seven specimens in AOM group are shown in Figure 8. In Figure 8(a), the mean storage modulus for control group was $16.31 \pm 2.31 \mathrm{MPa}$ at $0.2 \mathrm{kHz}$ and $22.35 \pm 2.57 \mathrm{MPa}$ at $8.0 \mathrm{kHz}$. The mean storage modulus for AOM group was $5.77 \pm 1.20 \mathrm{MPa}$ at $0.2 \mathrm{kHz}$ and $7.70 \pm 1.54 \mathrm{MPa}$ at $8.0 \mathrm{~Hz}$. Figure $8(\mathrm{~b})$ shows that the mean loss modulus from control group was $1.57 \pm 0.29 \mathrm{MPa}$ at $1.0 \mathrm{kHz}$ and reached a maximum of $4.44 \pm 0.71 \mathrm{MPa}$ at $5.5 \mathrm{kHz}$. The mean loss modulus from AOM group was $0.49 \pm 0.20 \mathrm{MPa}$ at $1.0 \mathrm{kHz}$ and $1.46 \pm 0.49 \mathrm{MPa}$ at $6.0 \mathrm{kHz}$. The mean loss modulus reached a maximum value at about $6.0 \mathrm{kHz}$.

Compared with the average complex modulus in control group, the decrease of storage modulus in AOM ears was about $65 \%$, and the decrease of loss modulus was about $32 \%$ over the frequency range of 0.2 to $8 \mathrm{kHz}$.

\section{Discussion}

\subsection{Dynamic property change of RWM in AOM ear}

In this study, the frequency dependent mechanical properties of the chinchilla RWM were determined by electromagnetic stimulation and FE modelling of the dynamic experiments. The mass effect of the bead was included in the FE model for simulation of the experiment. The mean resonance frequency of the RWM measured in AOM samples reduced from $1,478 \mathrm{~Hz}$ of control samples to $1,272 \mathrm{~Hz}$, which resulted in that the obtained complex modulus of normal specimen was significantly higher than that of AOM specimen. This result reflects the fact of AOM causing the change of material properties of the RWM. In addition to change of complex modulus, the structural properties in terms of flexural rigidity, mass, and geometric factor were also varied in 
AOM RWM. The flexural rigidity is expressed as $|D|=\frac{\left|E^{*}\right| h^{3}}{12\left(1-v^{2}\right)}$, the geometric factor of the specimen is characterised by the ratio of thickness to length of major /minor axis ( $a / b$ in this study) for an elliptical membrane. Using the mean data listed Tables 1 and 2, we calculated the average flexural rigidity of RWM in AOM ear and compared with that of normal ear and found that the mean flexural rigidity of RWM in AOM ears was about 1.2 times larger than normal ears across the frequency range of $200-8,000 \mathrm{~Hz}$. Note that the thickness of RWM in four day AOM ears increased by about $51 \%$ as displayed in the histological sections. The thickened RWM resulted in an increase of mass proportionally. Thus, the infectious RWM in AOM ears induced the increase of both mass and flexural rigidity of the tissue.

Figure 8 The mean complex modulus ( $E^{\prime}$ and $\left.E^{\prime \prime}\right)$ of six control RWM specimens and seven AOM specimens with standard deviations (SD), (a) storage modulus $E^{\prime}$, (b) loss modulus $E^{\prime \prime}$

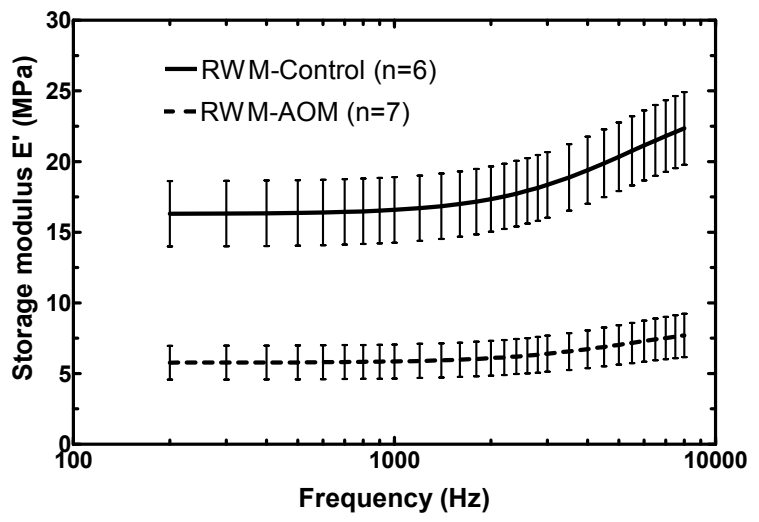

(a)

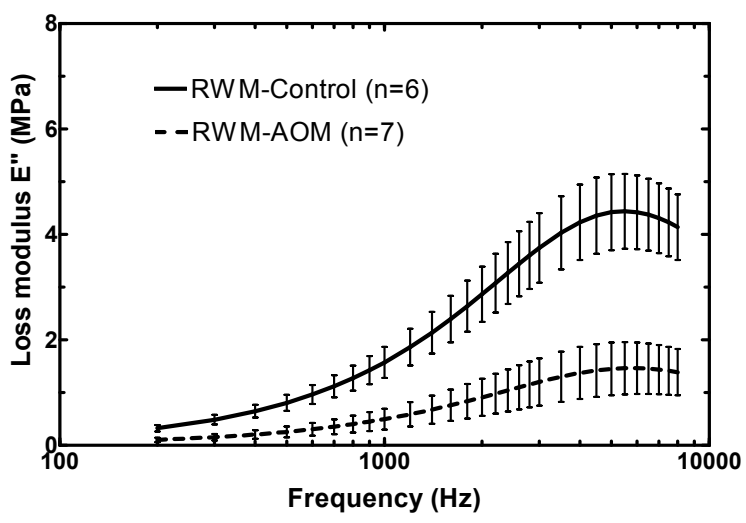

(b)

Assuming the mass of RWM increase of 51\% due to the thickened RWM and the RWM stiffness with the magnetic bead being constant, we used the Con-1 sample model to estimate the first order resonance frequency of this sample and found that the resonance 
frequency decreased only by $3.9 \%$. Similar results can also be obtained from other samples, which showed the mass-induced resonance frequency change. Comparing with the mean decrease of the resonance frequency in AOM ears by $13.94 \%$, which included contributions of mass, stiffness and geometric factor effects. We may conclude that the altered geometric factor result in the changes of the mass and strain energy distribution and reduce the resonance frequency in the AOM RWMs.

Figure 9 Comparison of the storage modulus $E^{\prime}$ and loss factor $\eta$ determined from the chichilla control samples in this study with those obtained from normal human RWMs with standard deviations (SD) (Zhang and Gan, 2013) and Guinea pig control samples (Gan et al., 2013), (a) storage modulus $E^{\prime}$, (b) loss factor $\eta$ (see online version for colours)

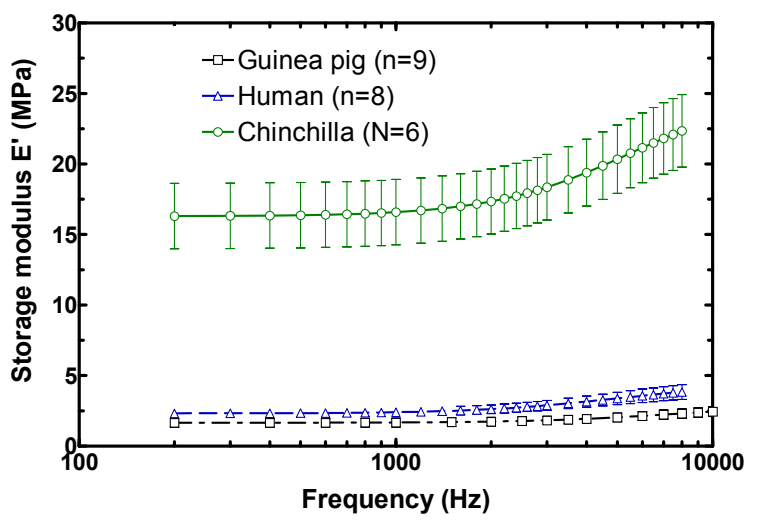

(a)

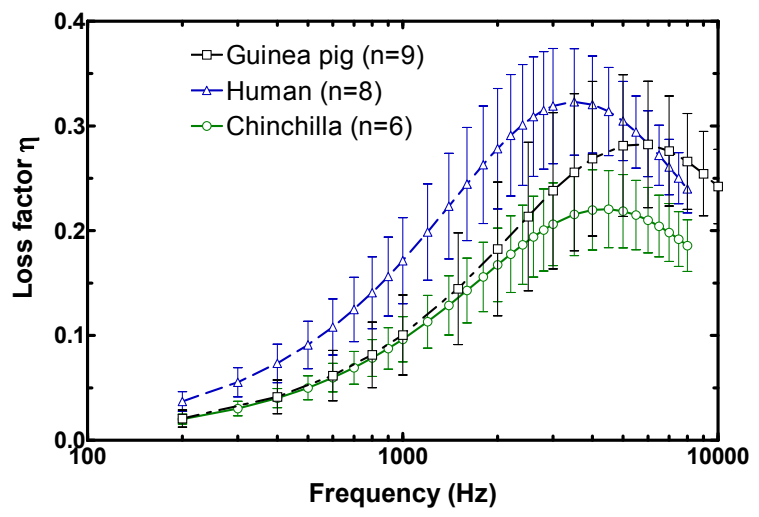

(b)

\subsection{Comparison with human and guinea pig RWM-control case}

RWMs of the human, guinea pig and chinchilla have comparable ultrastructures of three basic layers: an outer epithelial layer facing the middle ear, a middle fibrous layer, and an inner epithelium (Carpenter et al., 1989; Goycoolea and Lundman, 1997). Interspecies variations are mainly in thickness, being thinnest in chinchillas and thickest in humans. 
To quantitatively evaluate the dynamic parameters of the chinchilla RWM, we compared the results obtained in this study with the data of human RWMs and guinea pig RWMs reported by Zhang and Gan (2013) and Gan et al. (2013), respectively. Note that the human data were obtained from acoustic stimulation as the loading source.

The mean resonance frequency of guinea pig and chinchilla RWMs was $1,686 \mathrm{~Hz}$ and $1,478 \mathrm{~Hz}$, respectively. The mean resonance frequency of human RWM was $1,818 \mathrm{~Hz}$. Figures 9(a) and 9(b) show the comparison of storage modulus and loss factor over the frequency range of $0.2-8 \mathrm{kHz}$ among the human, guinea pig and chinchilla RWMs. The dashed lines with triangle symbols represent the results from human tissues, the dotted lines with square symbols display the results from guinea pig controls with cut off at $10 \mathrm{kHz}$, and the solid lines with circle symbols display the results from chinchilla controls. The storage modulus of chinchilla RWM is much higher than human and guinea pig: about 9.9 times larger than that of guinea pig at frequencies of $0.2-8 \mathrm{kHz}$, and 7.0 times larger than that of human at $0.2 \mathrm{kHz}$, and 5.8 times at $8 \mathrm{kHz}$. The loss factor of chinchilla RWM is the lowest among the human, guinea pig and chinchilla. The dynamic modulus of RWM of guinea pig, chinchilla and human has significant difference, but their resonance frequency range approximately from 1.5 to $1.8 \mathrm{kHz}$. This suggests that the largest dynamic compliance of the RWM for the three species occurs within the similar frequency region.

\subsection{Comparison with guinea pig RWM-AOM case}

Dynamic properties of the infectious RWM in AOM ears were measured first time by Gan et al. (2013) in guinea pigs. The results from nine guinea pig RWMs in AOM ears showed that the mean resonance frequency of the RWM with a magnet of $0.03 \mathrm{mg}$ decreased by $13.88 \%$, which was almost the same as that of chinchilla RWMs measured in this study. The mean resonance frequency decreased by $13.94 \%$ in the AOM chinchilla RWMs. Figures 10(a) and 10(b) show the comparisons of storage modulus and loss factor over the frequency range of $0.2-8 \mathrm{kHz}$ between the guinea pig and chinchilla RWMs. The dot dash lines with triangles display the results from guinea pig AOM ears with cut off at $10 \mathrm{kHz}$, and the solid lines display the results from chinchilla AOM ears. The storage modulus of chinchilla RWM in AOM ears is much higher than that of guinea pig. The largest difference of storage modulus between chinchilla and guinea pig is 17.2 times occurring at $0.2 \mathrm{kHz}$; the smallest difference is 15.8 time at $8.0 \mathrm{kHz}$. As can be seen in Figure 10(b), the loss factor of guinea pig RWM is still higher than that of chinchilla RWM.

Compared with the normal ears, the average decrease of the storage modulus in the guinea pig RWM of AOM ears ranged from $78 \%$ to $88 \%$ (Gan et al., 2013). In this study, the average decrease of the storage modulus in chinchilla AOM ears was about $65 \%$. This decrease in a relative value of the storage modulus in guinea pig is slightly larger than that in chinchilla. In contrast to the decrease in storage modulus, the bending rigidity of the guinea pig RWM in AOM ears was also larger than that of normal ears. From these two animal models of AOM, the shift in storage modulus reflects a 'weakening' in material property of RWM based on the isotropic, homogeneous material model, and the increase bending rigidity indicates a strengthening in the structural stiffness of RWM associated with AOM. 
Figure 10 Comparison of the storage modulus $E^{\prime}$ and loss factor $\eta$ determined from the chinchilla AOM samples in this study with those obtained from guinea pig AOM samples with standard deviations (SD) (Gan. et al., 2013), (a) storage modulus $E^{\prime}$, (b) loss factor $\eta$ (see online version for colours)

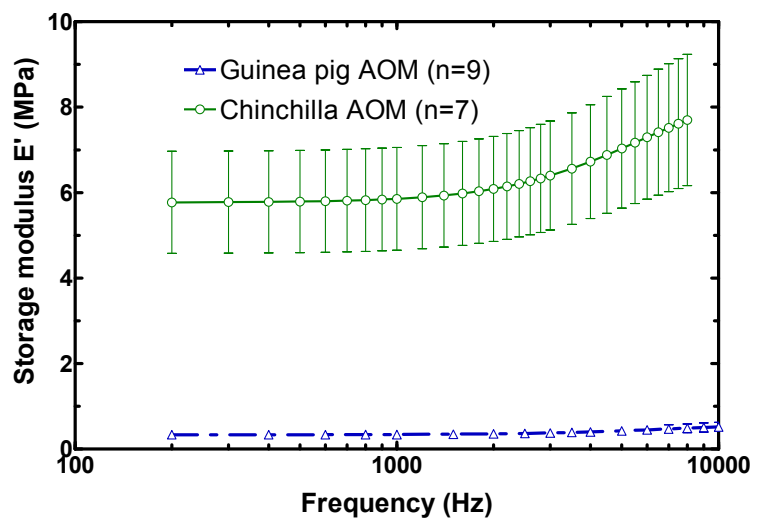

(a)

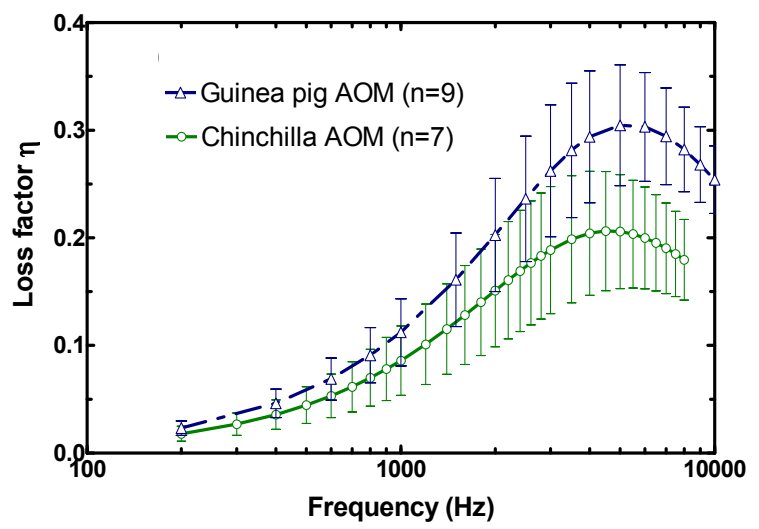

(b)

\subsection{Additional discussion of measurement and future study}

In this study, the RWM was simulated as a membrane structure with variable thickness and the magnetic bead was fixed on the membrane surface without detachment. However, the real contact status between the bead and membrane is hard to detect and can vary with the samples. To estimate the effect of contact status on complex modulus value of the RWM, we did the following two simulations:

1 A flexible layer representing the mucosal tissue between the bead and RWM was introduced to the FE model based on specimen OM-7. The flexible layer was assumed to be $5 \mu \mathrm{m}$ in thickness. The contact condition between the bead and membrane was simulated with varying elastic modulus of the flexible layer. When elastic modulus of the flexible layer was changed from 6.0 to $0.6 \mathrm{MPa}$ (about one-tenth of the mean storage modulus) and other parameters were maintained as the same as the original values for specimen OM-7, the result showed that the complex 
modulus value derived from the model was increased about $10 \%$ compared with the result from an ideal contact without detachment.

2 We estimated the effect of contact area change on the complex modulus. If $20 \%$ of the bead area was detached from the membrane along the perimeter of the bead, the complex modulus value obtained from the model was increased about $20 \%$. When the detached area increased by $50 \%$ of the bead surface, the complex modulus obtained from the model was increased about $50 \%$. These two simulations suggest that the perfect contact condition between the bead and membrane surface may cause an underestimation of the complex modulus value.

Moreover, this study was focused on the vibration induced at the centre of the RWM sample to derive the dynamic properties of the tissue, A LDV was used for experiment measurement. At high frequency, the RWM motion may become complicated and the measurement of multiple points on the RWM surface will probably provide more accurate data for calculating the dynamic properties of the RWM. However, the real answer to this approach will be part of our future study. In addition, a simultaneous determination of RWM mechanical properties and the quantitative description of the morphological features of the membrane may provide valuable information toward understanding the causes of stiffness change. An improved technology combining the morphological features into determination of mechanical properties of the tissue will be considered in the future study.

\section{Conclusions}

In this study, dynamic responses of the RWM specimens induced by electromagnetic force stimulation were measured by LDV over the frequency range of $0.2-8 \mathrm{kHz}$. Mechanical properties of the RWM were derived by the inverse-problem solving method and presented as the complex modulus.

The results obtained from this study show that the storage modulus and loss modulus of infectious RWM were both lower than those of normal RWM across the frequency range of 0.2 to $8 \mathrm{kHz}$. The average decrease of storage modulus in AOM ears was about $65 \%$ and that of loss modulus was about $32 \%$. In contrast, the derived bending rigidity of RWM in AOM ear was larger than that of control ear due to the $51 \%$ increase in thickness. The occurrence of the significant changes in dynamic modulus of the RWM in AOM ears was similar to previous measurements in guinea pigs. Our findings suggest that such alteration is common among the different animal models of AOM. The changes of complex modulus and bending rigidity in AOM ears provide insight into the effect of $\mathrm{AOM}$ on ear tissues from the mechanical standpoint.

\section{Acknowledgements}

The authors thank Xiying Guan and Shangyuan Jiang, graduate students in Biomedical Engineering Lab at the University of Oklahoma, for their technical assistance in histology studies. The authors also thank Dr. Xiangming Zhang, former graduate student for the measurement assistance on dynamic experiments. This work was supported by NIH R01DC011585 grant. 


\section{References}

Browning, G.C. and Granich, M.S. (1978) 'Surgical anatomy of the temporal bone in the Chinchilla', Annals of Otology, Rhinology, and Laryngology, Vol. 87, No. 6, pp.875-882.

Carpenter, A.M., Muchow, D. and Goycoolea, M.V. (1989) 'Ultrastructural studies of the human round window membrane', Archives of Otolaryngology-Head and Neck Surgery, Vol. 115, No. 5, pp.585-590.

Ethiraj, R. (2003) Bench Testing and Computer Modeling of Electromagnetic Coupling for a Hearing Implantable Device, Master thesis, University of Oklahoma, USA.

Gan, R.Z., Don, N. and Zhang, X. (2013) 'Dynamic properties of round window membrane in guinea pig otitis media model measured with electromagnetic stimulation', Hearing Research, Vol. 301, pp.125-136.

Goycoolea, M.V. (1995) 'Oval and round window membrane changes in otitis media in the human', Acta Otolaryngologica, (Stockh), Vol. 115, No. 2, pp.282-285.

Goycoolea, M.V. and Lundman, L. (1997) 'Round window membrane. Structure function and permeability: a review', Microscopy Research and Technique, Vol. 36, No. 3, pp.201-211.

Goycoolea, M.V., Muchow, D., Martinez, G.C., Goycoolea, H.G., Goycoolea, C.V., Schachern, P. and Knight, W. (1988) 'Permeability of the human round-window membrane to cationic ferritin', Archives of Otolaryngology-Head and Neck Surgery, Vol. 114, No. 11, pp.1247-1251.

Guan, X.Y., Chen, Y.Z. and Gan, R.Z. (2014) 'Factors affecting loss of tympanic membrane mobility in acute otitis media model of chinchilla', Hearing Research, Vol. 309, pp.136-146.

Ishii, T., Takayama, M. and Takahashi, Y. (1995) 'Mechanical properties of human round window, basilar and Reissner's membranes', Acta Otolaryngologica, Supplementum, Vol. 115, No. s519, pp78-82.

Machiraju, C., Phan, A.V., Pearsall, A.W. and Madanagopal, S. (2006) 'Viscoelastic studies of human subscapularis tendon: relaxation test and a weichert model', Computers Methods Programs in Biomedicine, Vol. 83, No. 1, pp.29-33.

Saber, A., Laurell, G., Bramer, T., Edsman, K., Engmer, C. and Ulfendahl, M. (2009) 'Middle ear application of a sodium hyaluronate gel loaded with neomycin in a guinea pig model', Ear \& Hearing, Vol. 30, No. 1, pp.81-89.

Sahni, R.S., Paparella, M.M., Schachern, P.A., Goycoolea, M.V. and Le, C.T. (1987) 'Thickness of the human round window membrane in different forms of otitis media', Archives of Otolaryngology-Head and Neck Surgery, Vol. 113, No. 6, pp.630-634.

Schachern, P., Tsuprun, V., Cureoglu, S., Ferrieri, P., Briles, D., Paparella, M. and Juhn, S. (2008) 'The round window membrane in otitis media: effect of pneumococcal proteins', Archives of Otolaryngology-Head and Neck Surgery, Vol. 134, No. 6, pp.658-662.

Vrettakos, P.A., Dear, S.P. and Saunders, J.C. (1988) 'Middle ear structure in the chinchilla: a quantitative study', American Journal of Otolaryngology, Vol. 9, No. 2, pp.58-67.

Wever, E.G. and Lawrence, M. (1948) 'The function of the round window', Annals of Otology, Rhinology, and Laryngology, Vol. 57, No. 3, pp.579-589.

Yoon, Y.J. and Hellstrom, S. (2002) 'Ultrastructural characteristics of the round window membrane during pneumococcal otitis media in rat', Journal Korean Medical Science, Vol. 17, No. 2, pp.230-235.

Zhang, X. and Gan, R.Z. (2013) 'Dynamic properties of human round window membrane in auditory frequencies', Medical Engineering \& Physics, Vol. 35, No. 3, pp.310-318, DOI: 10.1016/j.medengphy.2012.05.003. 